\title{
N-terminal residues of an HIV-1 gp41 membrane-proximal external region antigen influence broadly neutralizing 2 F5-like antibodies
}

\author{
Dezhi Li ${ }^{1,2 \#}$, Jie Liu ${ }^{2 \#}$, Li Zhang ${ }^{2}$, Tianshu $X^{2,3}$, Junheng Chen ${ }^{2,3}$, Liping Wang ${ }^{3 凶}$, Qi Zhao ${ }^{2,4 \bowtie ~}$ \\ 1. School of Life Sciences, Xiamen University, Fujian 361102, China \\ 2. Laboratory of Fully Human Antibody Engineering, Shenzhen Institutes of Advanced Technology, \\ Chinese Academy of Sciences, Guangdong 518055, China \\ 3. School of Life Sciences, Jilin University, Changchun 130012, China \\ 4. Faculty of Health Sciences, University of Macau, Macau, China
}

The Human immunodeficiency virus type 1 (HIV-1) gp41 membrane proximal external region (MPER) is targeted by broadly neutralizing antibodies (e.g. 2F5, 4E10, Z13e and m66.6), which makes this region a promising target for vaccine design. One strategy to elicit neutralizing antibodies against the MPER epitope is to design peptide immunogens mimicking neutralization structures. To probe 2F5-like neutralizing antibodies, two yeast-displayed antibody libraries from peripheral blood mononuclear cells from a HIV-1 patient were screened against the 2F5 epitope peptide SP62. Two 2F5-like antibodies were identified that specifically recognized SP62. However, these antibodies only weakly neutralized HIV-1 primary isolates. The epitopes recognized by these two 2F5-like antibodies include not only the 2F5 epitope (amino acids (aa) 662-667 in the MPER) but also several other residues (aa 652-655) locating at the $\mathrm{N}$-terminus in SP62. Experimental results suggest that residues of SP62 adjacent to the 2F5 epitope influence the response of broadly neutralizing 2F5-like antibodies in vaccination. Our findings may aid the design of vaccine immunogens and development of therapeutics against HIV-1 infection.

\section{KEYWORDS HIV-1; membrane proximal external region (MPER); 2F5; neutralizing antibody; yeast display}

\section{INTRODUCTION}

Human immunodeficiency virus type 1 (HIV-1) can rapidly generate mutants and evade immune responses (Vider-Shalit et al., 2009). This is the major obstacle to the development of prophylactic HIV-1 vaccines. Design of HIV-1 vaccine immunogens that can elicit high-titer,

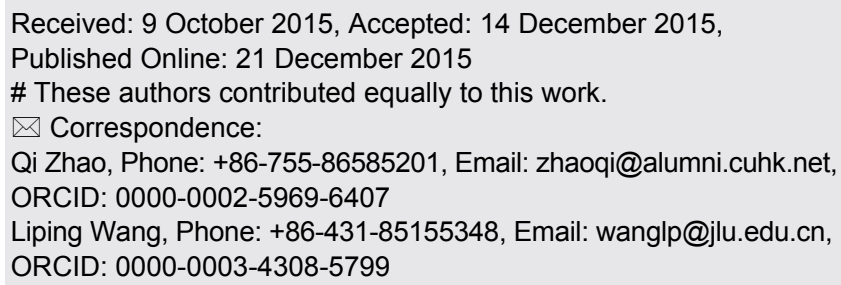

potent, broadly neutralizing antibodies (bnAbs) that inhibit viruses from different genetic subtypes is widely agreed (Liao et al., 2006; Zhang et al., 2008). Most of bnAbs primarily bind to membrane proximal external region (MPER) of glycoprotein 41 (gp41), glycans or CD4 binding site (Eroshkin et al., 2014). Several identified bnAbs, such as b12 (Roben et al., 1994), X5 (Moulard et al., 2002), 2G12 (Trkola et al., 1996), 2F5 and 4E10 (Stiegler et al., 2001; Zwick et al., 2001), exhibit potent, broad HIV-1 neutralizing activity in vitro and can prevent HIV-1 infection in animal models. These bnAbs target HIV-1 envelope glycoproteins (Envs) that are crucial for virus-cell fusion. Therefore, various Envs are potential candidate immunogens and have been evaluated in animal models and human clinical trials (Trkola et al., 
1996; Stiegler et al., 2001; Zwick et al., 2001; Moulard et al., 2002). However, failure to elicit bnAbs by using these Envs as immunogens reflects the difficulty to produce a vaccine that is effective in humans (Mehandru et al., 2004). It has been suggested that extensive characterization of bnAb-binding epitopes will help in the design of effective vaccine immunogens that are able to elicit bnAbs or similar antibodies in vivo (Alam et al., 2007). Although this approach is being pursued vigorously, few HIV-1 immunogens have efficiently produced neutralizing antibodies with broad specificity. For example, no vaccine or immunization strategy has been found to induce robust bnAb responses against gp41 MPER. A major aim of HIV-1 vaccine research is to determine how such rare bnAb responses are elicited.

The gp41 is a subunit of the HIV-1 Env complex and plays an important role in cell entry. The MPER is one of the most highly conserved sequences of gp41 that is crucial to the fusion of the viral and cellular membranes. MPER is a target for the neutralizing monoclonal antibodies 2F5, 4E10, Z13 and m66.6, making it a considerably interested epitope for HIV-1 vaccine development. The 2F5 epitope localizes on MPER spreading from amino acids (aa) 662 to 667 (sequence ELDKWA) (Montero et al., 2012), and that of m66.6 spans the 2F5 epitope and two additional upstream residues (L660 and L663) (Zhu et al., 2011). However, m66.6 exhibits less potency and narrower specificity than $2 \mathrm{~F} 5 \mathrm{bnAb}$. The existence of bnAbs such as 2F5 has fueled the hope that the development of efficacious HIV vaccines is achievable using immunogens comprising the epitopes recognized by these bnAbs (Chen et al., 2010). Unfortunately, failure so far to induce a high-titer neutralizing response in vaccination with antigenic envelope constructs expressing the 2F5 epitope reflects a lack of understanding of the mechanism of 2F5-like antibody evolution (Prabakaran et al., 2007; Shen et al., 2009). There is only sparse information about specific patients from whom HIV-1 bnAb specificities have been obtained. Therefore, the finding of novel bnAbs and their conserved epitopes still has implications for the design of HIV-1 vaccine immunogens and is also useful for understanding the mechanisms of HIV entry into host cells and evasion of immune responses.

In this study, we describe the identification and characterization of two human 2F5-like antibodies against the MPER antigen in yeast display antibody libraries constructed from a HIV-infected subject. The library was screened against peptide SP62 containing the 2F5 epitope. The identified 2F5-like antibodies weakly neutralized entry of virions pseudotyped with Envs from HIV-1 primary isolates. We speculate that the presence of residues aa ${ }_{652-655}$ adjacent to the $2 \mathrm{~F} 5$ epitope influences the immune response of eliciting neutralizing antibodies like 2F5. These data will be useful in the design of
MPER specific vaccine immunogens capable of eliciting bnAbs in vivo.

\section{MATERIALS AND METHODS}

\section{Cell lines, peptides, and antibodies}

Escherichia coli (E. coli) TOP10 was used for cloning and preparation of plasmid DNA. E. coli HB2151 was used for expression of soluble single-chain fragment variable antibody ( $\mathrm{scFv}$ ) and single chain Fab antibody (scFab). HEK293T was purchased from ATCC. Other cell lines and plasmids for expression of various HIV-1 Envs were obtained from the National Institutes of Health AIDS Research and Reference Reagent Program (ARRRP). Three biotinylated peptides, SP62 (QQEKNEQELLELDKWASLWN), SCR (the amino acid sequence of SP62 scrambled), and MPER656 (NEQELLELDKWASLWNWFNITNWLW), were synthesized by Genscript. Horseradish peroxidase (HRP)conjugated anti-FLAG antibody and HRP-conjugated anti-human $\operatorname{IgG}$ (Fc-specific) antibody were purchased from Sigma-Aldrich. Alexa-488 goat anti-mouse IgG antibody, mouse anti-c-Myc antibody and streptavidin Rphycoerythrin conjugate were obtained from Invitrogen.

\section{Yeast library construction}

cDNA was reverse transcribed from total RNA of peripheral blood monouclear cells isolated from a HIV-1 infected patient. Heavy- and light-chain of antibody genes were PCR amplified from cDNA using a primer set based on the protocol of Zhu and Dimitrov (Zhu and Dimitrov, 2009). The construction of $\mathrm{scFv}$ and $\mathrm{scFab}$ genes was as described previously (Walker et al., 2009). The preparation of yeast libraries was performed according to our previous publications (Zhao et al., 2012; Zhao et al., 2015). The size of the scFv and scFab libraries was estimated at $4 \times 10^{6}$ and $1 \times 10^{6}$ members, respectively.

\section{Library selection and flow cytometric analysis}

Construction and culture of yeast libraries for affinity maturation were as described previously with some modifications (Zhao et al., 2011; Zhao et al., 2012). Briefly, yeast cells were grown in SD-CAA media, and induced in SG-CAA in volumes appropriate for the size of the library (Chao et al., 2006). For library selection, induced yeast cells $\left(1 \times 10^{9}\right)$ were incubated in $3 \mathrm{~mL}$ of PBSA buffer $(0.1 \%$ BSA in PBS) with $1 \mu \mathrm{g} / \mathrm{mL}$ peptide SP62 for $1 \mathrm{~h}$ at $4{ }^{\circ} \mathrm{C}$ with gentle rotation. Excess peptides were washed away in cold PBSA, and then cells were mixed with $50 \mu \mathrm{L}$ Dynabeads MyOne Streptavidin T1 beads (Invitrogen) in $3 \mathrm{~mL}$ PBSA buffer. Capture was performed at $4{ }^{\circ} \mathrm{C}$ for $1 \mathrm{~h}$ with gentle rotation. A magnet was then applied to select out the beads, and the supernatants were removed. After washing 3 times with 
ice-cold PBSA buffer, the cell pellet was cultured in 10 $\mathrm{mL}$ SD-CAA media at $30^{\circ} \mathrm{C}$ overnight. The yeast cells recovered from the magnetic bead were induced in 50 $\mathrm{mL}$ SG-CAA for $16-18 \mathrm{~h}$ at $20^{\circ} \mathrm{C}$. The following two to three rounds of sorting were performed by repeating the above steps. After the final round of selection, yeast cells were cultured on SD-CAA plates and individual colonies were picked for characterization.

Binding of yeast cells was analyzed using flow cytometry. Typically, $1 \times 10^{6}$ cells were stained in $50 \mu \mathrm{L}$ of PBS in the presence of $0.1 \mu \mathrm{g} / \mathrm{mL}$ biotinylated peptide and $5 \mu \mathrm{g} / \mathrm{mL}$ mouse anti-c-Myc antibody with $30 \mathrm{~min}$ incubation and washed with $200 \mu \mathrm{L}$ PBSA buffer. For competition analysis, $2 \mathrm{~F} 5 \mathrm{IgG}$ was pre-incubated with peptides and then incubated with cells for $30 \mathrm{~min}$ at $4{ }^{\circ} \mathrm{C}$. Yeast cells were washed and then incubated with 5 $\mu \mathrm{g} / \mathrm{mL}$ Alexa-488 goat anti-mouse IgG antibody (Invitrogen) and $10 \mu \mathrm{g} / \mathrm{mL}$ streptavidin R-phycoerythrin conjugate. The cells were resuspended in PBSA buffer and counted using a BD FACSCalibu ${ }^{\mathrm{TM}}$ Flow Cytometer.

\section{Expression and purification of antibodies}

Isolation of plasmid DNA from individual yeast cells was performed using the Zymoprep Yeast Plasmid Miniprep Kit II (Zymo Research). The plasmids were then transformed into competent E.coli TOP10 cells for amplification of plasmid DNA which was purified using the QIAprep Spin Miniprep Kit (Qiagen). Individual DNA inserts were obtained from plasmids digested with the restriction enzyme $S f i$ I and ligated into similarly digested vector pComb3X for soluble expression in E. coli HB2151. scFv and scFab were expressed and purified as previously described (Zhao et al., 2009). The supernatant was used for purification of $\mathrm{scFv}$ and $\mathrm{scFab}$ by immobilized metal ion affinity chromatography (IMAC) using Ni-NTA resin (Qiagen) according to the manufacturer's protocols.

\section{ELISA}

Various concentrations of antigens were diluted in PBS buffer and coated onto 96-well ELISA plates at 4 ${ }^{\circ} \mathrm{C}$ overnight. The plate was then blocked with PBS containing 3\% powdered milk buffer. Antibodies were serially diluted in the same blocking buffer and applied to the 96-well ELISA plates. Mouse anti-Flag-HRP was used to detect Flag tag at the C-terminal end of each of the scFvs or scFabs, and mouse anti-human Fc-HRP was used to detect Flag tag. The 2,2'-azinobis [3-ethylbenzothiazoline-6-sulfonic acid]-diammonium salt (ABTS) solution was then added to each well and optical density at $450 \mathrm{~nm}\left(\mathrm{OD}_{450}\right)$ was measured $10 \mathrm{~min}$ later.

\section{Pseudovirus neutralization assay}

Viruses pseudotyped with HIV-1 Envs were prepared by cotransfection of $70 \%-80 \%$ confluent $293 \mathrm{~T}$ cells with
pNL4-3.luc. $\mathrm{E}^{-} \mathrm{R}^{-}$and $\mathrm{pSV7d}$ constructs encoding HIV-1 Envs by using the PolyFect transfection reagent (Qiagen), according to the manufacturer's instructions. Pseudotyped viruses were obtained after $48 \mathrm{~h}$ by centrifugation and filtration of cell culture through $0.45-\mu \mathrm{m}$ filters. For neutralization assays, viruses were mixed with different concentrations of antibodies for $1 \mathrm{~h}$ at 37 ${ }^{\circ} \mathrm{C}$, and then the mixture was added to about $1.5 \times 10^{4}$ HOS-CD4-CCR5 (used for all R5 and dual tropic viruses) cells grown in each well of 96-well plates. Luminescence was measured after $48 \mathrm{~h}$ using the Bright-Glo Luciferase Assay System (Promega) and a LumiCount microplate luminometer (Turner Designs). Mean relative light units (RLU) were determined for duplicate wells. Relative infectivity (\%) was calculated by the following formula: (average RLU of antibody-containing wells/average RLU of virus-only wells) $\times 100$.

\section{RESULTS}

\section{Generation of scFv and scFab yeast libraries}

The plasmid used for library construction, pYD7, was modified from pCTCON2 (Hackel et al., 2008). Two Sfi I restriction sites at both ends of the scFv DNA fragment in pYD7 matched the cloning sites in the pComb3X vector. This allowed fragments to be shuttled between the yeast vector and pComb3X. The linkers $\left(\mathrm{G}_{4} \mathrm{~S}\right)_{3}$ and $(\mathrm{SGGG})_{2}(\mathrm{SEGGG})_{4}(\mathrm{SGGGSG})$ (Walker et al., 2009) were inserted between the heavy and light chains of antibody, generating $\mathrm{scFv}$ and $\mathrm{scFab}$ respectively. The $\mathrm{scFv}$ $\left(4 \times 10^{6}\right)$ and scFab $\left(1 \times 10^{6}\right)$ yeast libraries were constructed from peripheral blood B cells of an HIV-1 infected patient who exhibits broad serum neutralization. To estimate the expression of libraries, library yeast cells were stained with mouse anti-c-Myc antibody and then counted using flow cytometry. More than $40 \%$ of the yeast clones were expressed in the scFv library, but only around $10 \%$ of the yeast clones were expressed in the scFab library.

\section{Selection of yeast surface displayed ScFv and scFab libraries with MPER peptide}

The $\mathrm{scFv}$ and $\mathrm{scFab}$ libraries were subjected to multiple rounds of magnetic bead selection against MPER peptide SP62 according to the protocol of Yeung and Wittrup (Yeung and Wittrup, 2002). Significant enrichment was obtained from the scFv library after three rounds selection (Figure 1A), but enrichment was not obvious from the scFab library after four rounds selection (Figure 1B). Following the final round of selection, polyclonal yeast cells were cultured for characterization. The 2F5 IgG antibody was used to determine whether enriched clones bound the 2F5 epitope on MPER peptide SP62. Eight of 36 clones from the scFv displayed library were identified to bind to the SP62 peptide. Sequence 
A
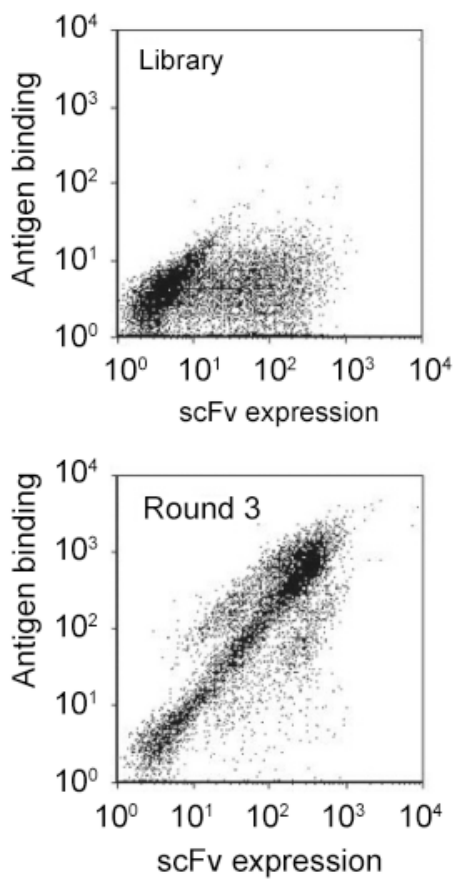

B
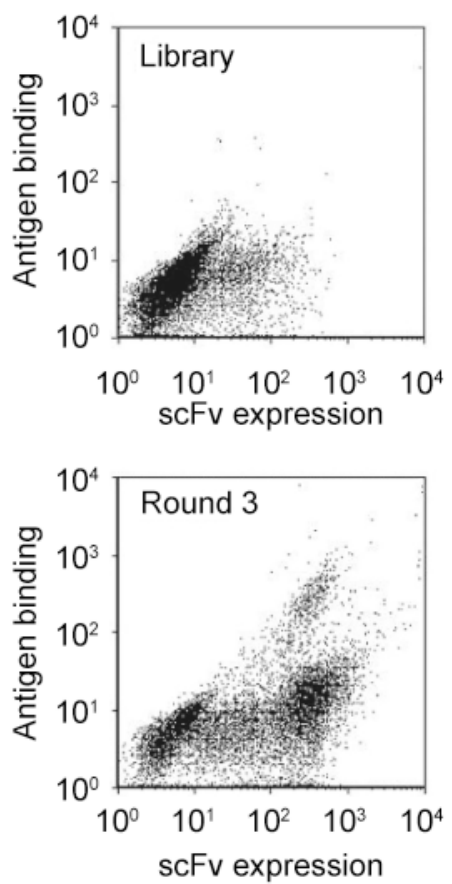
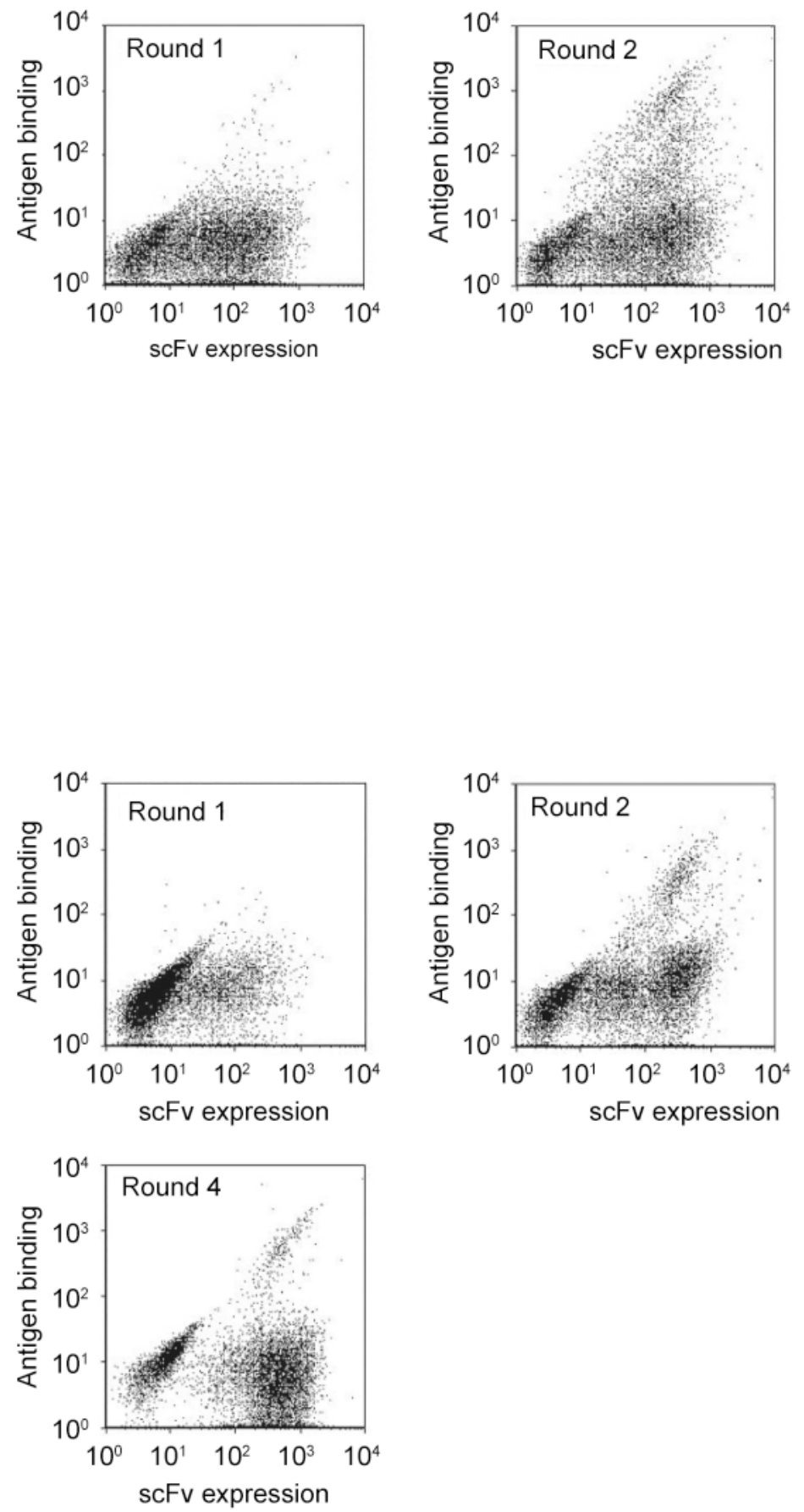

Figure 1. FACS analysis of $\mathrm{scFv}(\mathrm{A})$ and $\mathrm{scFab}(\mathrm{B})$ from yeast libraries for panning. Yeast cells were labeled with mouse anti-c-Myc antibody followed by goat anti-mouse dye, as well as biotinylated SP62 peptide followed by streptavidin-dye. Then, yeast cell were analyzed by flow cytometry. Yeast cells expressing scFv (A) and scFab (B) were selected for 3 and 4 rounds, respectively.

analysis of these eight clones showed that they represented two individual clones, 3A2a and 3A5a (Figure 2A, 2B), containing identical light chains of the IGKV1 family and very closely related heavy chains of the IGHV3 family.

To further characterize the selected antibodies (3A2a and $3 \mathrm{~A} 5 \mathrm{a}$ ), they were expressed as scFv fragments. ELISA showed that $3 \mathrm{~A} 2 \mathrm{a}$ and $3 \mathrm{~A} 5 \mathrm{a}$ specifically bound to MPER peptide SP62 with relatively high affinity $\left(\mathrm{EC}_{50}\right.$ values of $2 \mathrm{nmol} / \mathrm{L}$ and $7 \mathrm{nmol} / \mathrm{L}$ ) (Figure 2A, 2B), while no significant binding to scrambled SP62 peptide (SCR) was observed (Figure 2C, 2D). 
The selected antibodies weakly neutralized HIV-1 isolates

To investigate whether the antibodies were capable of neutralizing HIV-1 primary isolates, we used viruses pseudotyped with Envs from HIV-1 isolates JRFL and $\mathrm{Bal}$, representing clade B. Neither of the antibodies exhibited strong neutralizing activity (Figure 3). Both 3A2a and $3 \mathrm{~A} 5 \mathrm{a}$ antibodies exhibited weaker neutralizing activ-

A

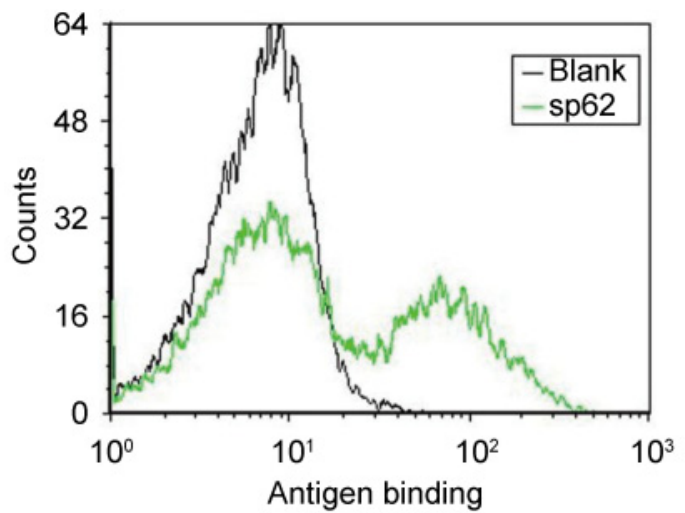

C

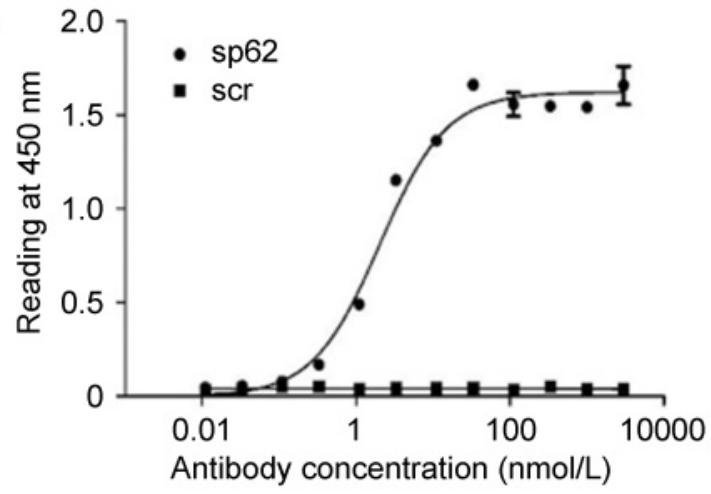

ity than m66 Fab. 3A2a and 3A5a could even enhance viral infection by JRFL when these antibodies were at high concentrations. The m66 antibody was previously identified as a bnAb 2F5-like antibody from an HIV-1 patient (Zhu et al., 2011). The positive control antibody 2F5 IgG showed efficient neutralization activity.

\section{Characterization of the antibody epitopes}

B
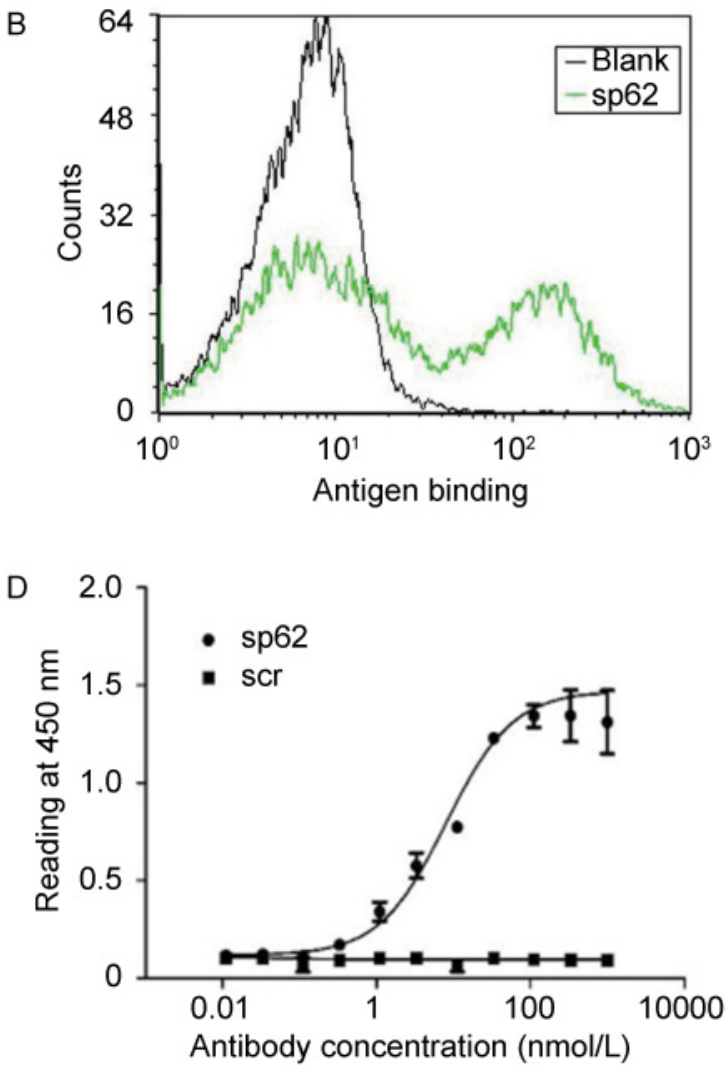

Figure 2. Specific binding of $3 A 2 a$ and $3 A 5 a$ scFvs to peptide SP62 (A, B). Yeast cells expressing 3A2a (A) and 3A5a (B) were treated with biotinylated SP62 peptide (green) followed by streptavidin-dye staining and analyzed by flow cytometry. The blank histogram indicates staining without antigen. Three-fold serially diluted 3A2a (C) and 3A5a (D) scFvs were added to 96-well plates coated with SP62 peptide $(\bullet)$ and control scrambled (SCR) peptide ( $\bullet$ ). Bound Abs were detected using HRP conjugated to anti-Flag antibody and 2,2'-azinobis [3-ethylbenzothiazoline-6-sulfonic acid]-diammonium salt (ABTS) as substrate.
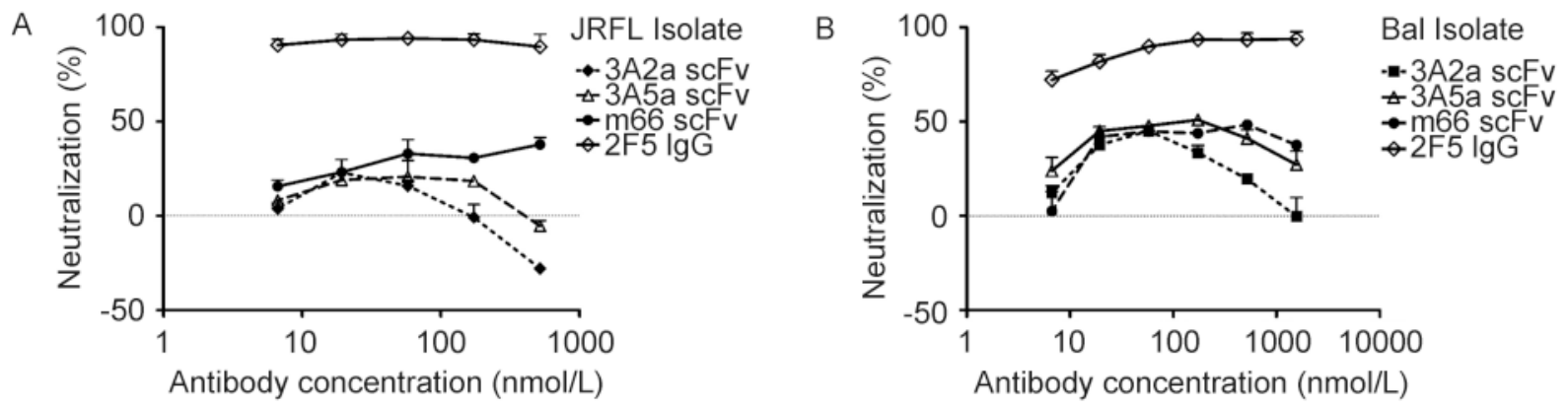

Figure 3. Neutralization assay of HIV-1 isolates JRFL (A) and Bal (B) in a pseudovirus/cell line by 3A2a and 3A5a. 2F5 IgG and $\mathrm{m} 66 \mathrm{scFv}$ treated samples were positive controls. 
The epitopes of four well-known broadly neutralizing MPER-specific antibodies, 2F5, 4E10, Z13 and m66.6, include the MPER of gp41. They bind to linear peptide sequences located in the MPER. To find out whether our isolated antibodies bound to the same epitope as 2F5 antibody, we performed competition flow cytometric assays between 3A5a-displayed yeasts and 2F5 antibody or a control antibody (m102.4) against Nipah virus and Hendra virus (Zhu et al., 2008). The yeast cells competitively decreased the binding of 2F5 antibody to MPER peptide SP62, but not that of the irrelevant (negative control) antibody m102.4 (Figure 4A). We also measured the binding of soluble scFv fragments of $3 \mathrm{~A} 5 \mathrm{a}$ in competition ELISA with $2 \mathrm{~F} 5$ antibody. 3A5a significantly blocked the binding of $2 \mathrm{~F} 5 \mathrm{IgG}$ to SP62 peptide (Figure 4B). These data implied that antibody $3 \mathrm{~A} 5 \mathrm{a}$ shared the same, or an overlapping, epitope with 2F5 antibody.

To further localize the antibody epitopes, we measured antibody binding to the MPER656 peptide that comprises the 2F5 and 4E10 epitopes but lacks several residues $\left(\mathrm{aa}_{652-655}\right)$ that are present at the $\mathrm{N}$-terminus of SP62 (Figure 5A). Both 3A2a and 3A5a bond SP62 but not MPER656 (Figure 5B). Thus, 3A2a and 3A5a lost binding activity on deletion of the sequence QQEK. Therefore, we suggest that the epitope of the weaklyneutralizing antibodies (mAbs) $3 \mathrm{~A} 2 \mathrm{a}$ and $3 \mathrm{~A} 5 \mathrm{a}$ requires the $2 \mathrm{~F} 5$ epitope $\left(\mathrm{aa}_{662-667}\right)$ and, additionally, gp41 $652-655$.

\section{DISCUSSION}

An effective HIV-1 vaccine should elicit broadly neutralizing antibodies targeting the HIV Envs, such as glycan shield, CD4 binding site, the coreceptor binding

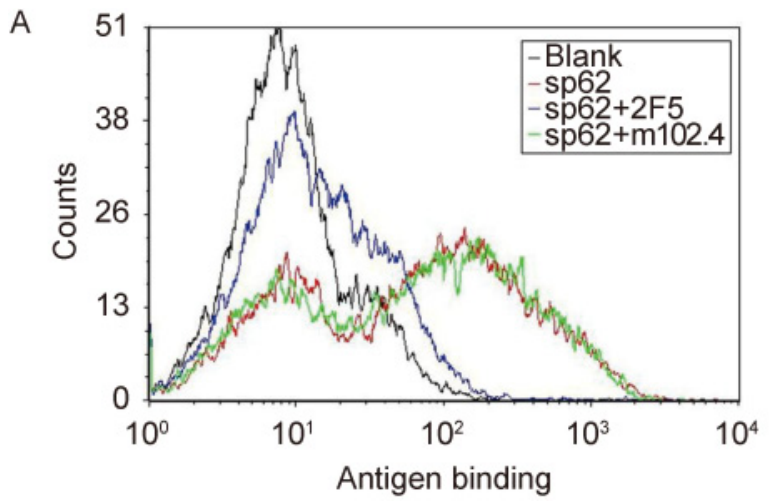

site, Env fusion intermediates, and MPER (Lin and Nara, 2007; Phogat and Wyatt, 2007). However, most of the HIV-1 vaccines based on unmodified Envs do not efficiently induce high-titer bnAbs to combat the highly variable viruses in human clinical trials.

In recent years, the very conserved gp41 MPER has gained attention as a new vaccine immunogen. However, the gp41 MPER epitopes are normally evanescent or poorly presented in vivo (Chen et al., 2010). In addition, the HIV-1 gp41 frequently induces non-neutralizing 2F5like antibodies in HIV-1 patients. This raises the question of how the binding properties of gp41 non-neutralizing antibodies differ from those effective bnAbs. It is important to stabilize and present the $2 \mathrm{~F} 5$ epitope in its proper structural configuration (Holl et al., 2014). The rational modification of MPER vaccines can prolong the exposure of the gp41-neutralizing epitopes, thus allowing for a longer time for MPER antibody binding.

In a previous report (Zhu et al., 2011), we described two 2F5-like antibodies (m66 and m66.6) that target the core 2F5 epitope (664DKW666) and two additional upstream residues (L660, L663). Neutralization of HIV-1 by $\mathrm{m} 66.6$ was more effective than that by $\mathrm{m} 66$, but weaker than that by $2 \mathrm{~F} 5$. In this study, we described two 2F5-like antibodies (3A2a and $3 \mathrm{~A} 5 \mathrm{a}$ ) targeting the highly conserved regions of gp41 which are in very close proximity to the $2 \mathrm{~F} 5$ binding site. The binding profile of $3 \mathrm{~A} 2 \mathrm{a}$ and $3 \mathrm{~A} 5 \mathrm{a}$ to MPER peptides was very similar to that of m66. All of them showed high affinity for the SP62 peptide. Similar to m66, we observed that the localization of two 2F5-like antibodies required the 2F5 epitope $\left(\mathrm{aa}_{662-667}\right)$ and several additional residues at the $\mathrm{N}$ terminus of SP62. The difference was that the epitopes of $3 \mathrm{~A} 2 \mathrm{a}$ and $3 \mathrm{~A} 5 \mathrm{a}$ involve $\mathrm{aa}_{652-655}$ whereas the epitope of

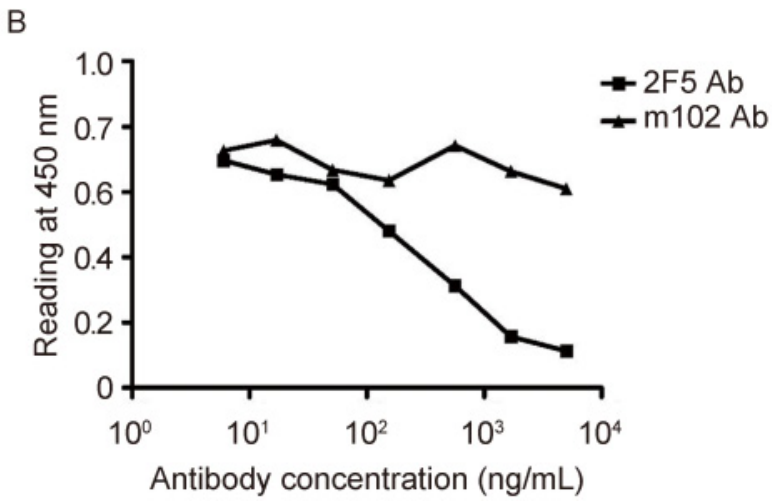

Figure 4. Competition assay of 3A5a scFv and 2F5 IgG for binding to SP62 peptide. (A) Enriched yeast cells from the scFv library were incubated with biotinylated SP62 peptide (red), a mixture of SP62 and 2F5 lgG (blue), or a mixture of SP62 and m102.4 lgG (green) and then stained with R-phycoerythrin conjugated streptavidin. Cells were detected by flow cytometry. (B) Mixtures of 3A5a scFv and 2F5 IgG (a) or control m102.4 IgG ( $\mathbf{\Delta}$ ) were added to 96-well plates coated with SP62 peptide. Bound Abs were detected using HRP conjugated to anti-Flag antibody and ABTS as substrate. 
A

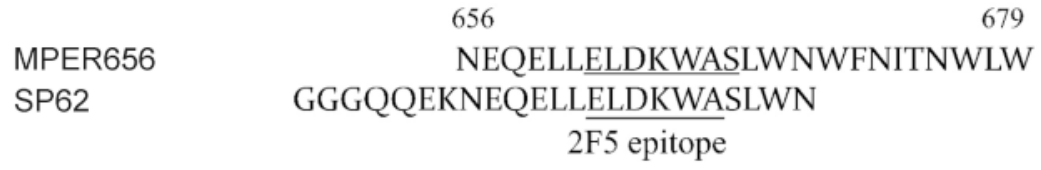

B

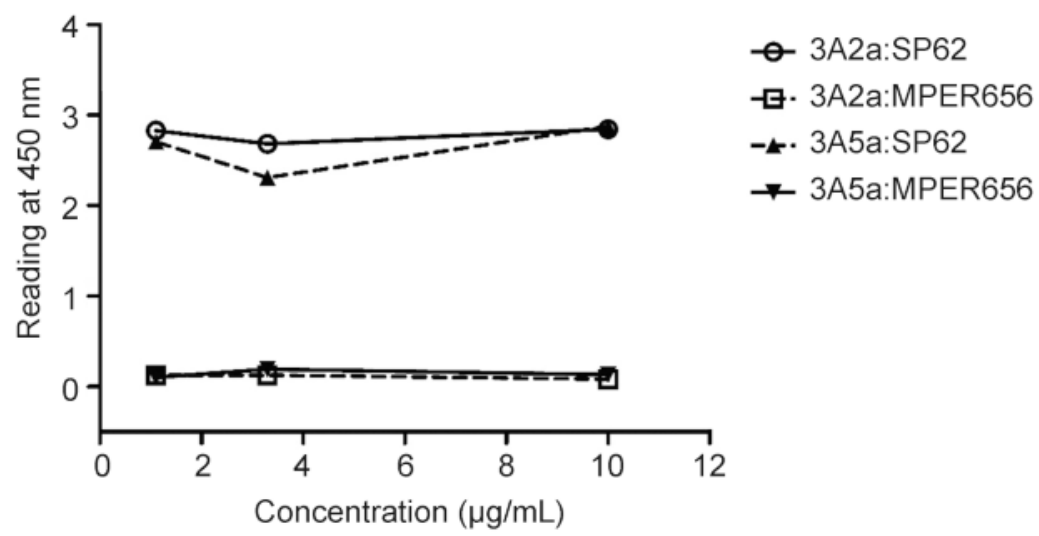

Figure 5. Epitope mapping of 3A2a and 3A5a scFvs against SP62 and MPER656 peptides. (A) Alignment of SP62 and MPER656 sequences. (B) ScFvs were added to 96-well plates coated with SP62 or MPER656 peptide. Bound Abs were detected using HRP conjugated to anti-Flag antibody and ABTS as substrate.

m66 involves $\mathrm{aa}_{660}$ and $\mathrm{aa}_{663}$. These results strongly indicate that the 3A2a and 3A5a epitope overlaps with that of $2 \mathrm{~F} 5$, but the two epitopes are different.

m66 was less effective than $2 \mathrm{~F} 5$ in a neutralization assay of HIV-1 primary isolates. Despite similarities to m66, the two 2F5-like antibodies identified here exhibited weaker HIV-1-neutralizing activity than m66. They gradually lost the ability to block the viral infection at high antibody concentration. We speculate that these antibodies might aggregate at high concentration due to low stability, resulting in non-specific binding to the cell surface. One could hypothesize that the possible high immunogenicity of the conserved non-neutralizing epitopes adjacent to the 2F5 epitope could further induce abundant non-neutralizing antibodies and shield neutralizing epitopes from immune system responses. In addition, these antibodies could directly prevent the detection of some neutralizing antibodies. Therefore, rational design of immunogens based on MPER would exclude the region $\mathrm{aa}_{652-657}$. These data also have important implications for reducing the immunogenicity of unwanted epitopes when the MPER region is used as an immunogen.

\section{ACKNOWLEDGMENTS}

This work was supported by the Natural Science Foundation of Guangdong (No. 2015A030313741), the National Natural Science Foundation of China (No. 31440041), Shenzhen Peacock Innovation Plan Fund (No. KQCX20140520154115029), Shenzhen Know- ledge Innovation Program (No. JCYJ20140901003939 026), and Novo Nordisk A/S - Chinese Academy of Sciences Research Fund (No. NNCAS-2013-9). We are grateful to Dr. Dimiter S. Dimitrov, National Cancer Insitute, NIH, for his expertise and advice.

\section{COMPLIANCE WITH ETHICS GUIDELINES}

The authors declare that they have no conflict of interest. This article does not contain any studies with human or animal subjects performed by any of the authors.

\section{AUTHOR CONTRIBUTIONS}

DL and JL conceived and executed experiments. TX conceived and executed experiments related to antibody purification. JC conceived of and executed experiments related to library screening. LW conceived and co-wrote the manuscript. QZ supervised all aspects of the investigation and co-wrote the manuscript.

\section{REFERENCES}

Alam SM, McAdams M, Boren D, Rak M, Scearce RM, Gao F, Camacho ZT, Gewirth D, Kelsoe G, Chen P, Haynes BF. 2007. The role of antibody polyspecificity and lipid reactivity in binding of broadly neutralizing anti-HIV-1 envelope human monoclonal antibodies 2 F5 and 4E10 to glycoprotein 41 membrane proximal envelope epitopes. J Immunol, 178: 4424-4435.

Chao G, Lau WL, Hackel BJ, Sazinsky SL, Lippow SM, Wittrup KD. 2006. Isolating and engineering human antibodies using yeast surface display. Nat Protoc, 1: 755-768. 
Chen WZ, Zhu ZY, Liao HX, Quinnan G, Broder CC, Haynes BF, Dimitrov DS. 2010. Cross-Reactive Human IgM-Derived Monoclonal Antibodies that Bind to HIV-1 Envelope Glycoproteins. Viruses 2: 547-565.

Eroshkin AM, LeBlanc A, Weekes D, Post K, Li Z, Rajput A, Butera ST, Burton DR, Godzik A. 2014. bNAber: database of broadly neutralizing HIV antibodies. Nucleic Acids Res, 42: D1133-D1139.

Hackel BJ, Kapila A, Wittrup KD. 2008. Picomolar affinity fibronectin domains engineered utilizing loop length diversity, recursive mutagenesis, and loop shuffling. J Mol Biol, 381: $1238-1252$.

Holl TM, Yang G, Kuraoka M, Verkoczy L, Alam SM, Moody MA, Haynes BF, Kelsoe G. 2014. Enhanced antibody responses to an HIV-1 membrane-proximal external region antigen in mice reconstituted with cultured lymphocytes. J Immunol, 192: 3269-3279.

Liao HX, Sutherland LL, Xia SM, Brock ME, Scearce RM, Vanleeuwen S, Alam SM, McAdams M, Weaver EA, Camacho Z, Ma BJ, Li Y, Decker JM, Nabel GJ, Montefiori DC, Hahn BH, Korber BT, Gao F, Haynes BF. 2006. A group M consensus envelope glycoprotein induces antibodies that neutralize subsets of subtype B and C HIV-1 primary viruses. Virology, 353: 268-282.

Lin G, Nara PL. 2007. Designing immunogens to elicit broadly neutralizing antibodies to the HIV-1 envelope glycoprotein. Curr HIV Res, 5: 514-541.

Mehandru S, Wrin T, Galovich J, Stiegler G, Vcelar B, Hurley A, Hogan C, Vasan S, Katinger H, Petropoulos CJ, Markowitz M. 2004. Neutralization profiles of newly transmitted human immunodeficiency virus type 1 by monoclonal antibodies $2 \mathrm{G} 12$, 2F5, and 4E10. J Virol, 78: 14039-14042.

Montero M, Gulzar N, Klaric KA, Donald JE, Lepik C, Wu S, Tsai S, Julien JP, Hessell AJ, Wang S, Lu S, Burton DR, Pai EF, Degrado WF, Scott JK. 2012. Neutralizing epitopes in the membrane-proximal external region of HIV-1 gp41 are influenced by the transmembrane domain and the plasma membrane. J Virol, 86: 2930-2941.

Moulard M, Phogat SK, Shu Y, Labrijn AF, Xiao X, Binley JM, Zhang MY, Sidorov IA, Broder CC, Robinson J, Parren PW, Burton DR, Dimitrov DS. 2002. Broadly cross-reactive HIV-1neutralizing human monoclonal Fab selected for binding to gp120-CD4-CCR5 complexes. Proc Natl Acad Sci U S A, 99: 6913-6918.

Phogat S, Wyatt R. 2007. Rational modifications of HIV-1 envelope glycoproteins for immunogen design. Curr Pharm Des, 13: 213-227.

Prabakaran P, Dimitrov AS, Fouts TR, Dimitrov DS. 2007. Structure and function of the HIV envelope glycoprotein as entry mediator, vaccine immunogen, and target for inhibitors. Adv Pharmacol, 55: 33-97.

Roben P, Moore JP, Thali M, Sodroski J, Barbas CF, 3rd, Burton DR. 1994. Recognition properties of a panel of human recombinant Fab fragments to the CD4 binding site of gp120 that show differing abilities to neutralize human immunodeficiency virus type 1. J Virol, 68: 4821-4828.

Shen X, Parks RJ, Montefiori DC, Kirchherr JL, Keele BF, Decker JM, Blattner WA, Gao F, Weinhold KJ, Hicks CB, Greenberg ML, Hahn BH, Shaw GM, Haynes BF, Tomaras GD. 2009. In vivo gp41 antibodies targeting the $2 \mathrm{~F} 5$ monoclonal antibody epitope mediate human immunodeficiency virus type 1 neutralization breadth. J Virol, 83: 3617-3625.

Stiegler G, Kunert R, Purtscher M, Wolbank S, Voglauer R, Steindl F, Katinger H. 2001. A potent cross-clade neutralizing human monoclonal antibody against a novel epitope on gp41 of human immunodeficiency virus type 1. AIDS Res Hum Retro- viruses, 17: 1757-1765.

Trkola A, Purtscher M, Muster T, Ballaun C, Buchacher A, Sullivan N, Srinivasan K, Sodroski J, Moore JP, Katinger H. 1996. Human monoclonal antibody $2 \mathrm{G} 12$ defines a distinctive neutralization epitope on the gp120 glycoprotein of human immunodeficiency virus type 1. J Virol, 70: 1100-1108.

Vider-Shalit T, Almani M, Sarid R, Louzoun Y. 2009. The HIV hide and seek game: an immunogenomic analysis of the HIV epitope repertoire. AIDS, 23: 1311-1318.

Walker LM, Bowley DR, Burton DR. 2009. Efficient recovery of high-affinity antibodies from a single-chain Fab yeast display library. J Mol Biol, 389: 365-375.

Yeung YA, Wittrup KD. 2002. Quantitative screening of yeast surface-displayed polypeptide libraries by magnetic bead capture. Biotechnol Prog, 18: 212-220.

Zhang MY, Vu BK, Choudhary A, Lu H, Humbert M, Ong H, Alam M, Ruprecht RM, Quinnan G, Jiang S, Montefiori DC, Mascola JR, Broder CC, Haynes BF, Dimitrov DS. 2008. Crossreactive human immunodeficiency virus type 1-neutralizing human monoclonal antibody that recognizes a novel conformational epitope on gp41 and lacks reactivity against self-antigens. J Virol, 82: 6869-6879.

Zhao Q, Ahmed M, Guo H, Cheung IY, Cheung NK. 2015. Alteration of electrostatic surface potential enhances affinity and tumor killing properties of anti-GD2 monoclonal antibody hu3F8. J Biol Chem, 290: 13017-13027.

Zhao Q, Ahmed M, Tassev DV,Hasan A, Kuo T, Guo H, O'Reilly RJ, Cheung NK. 2015. Affinity maturation of T-cell receptorlike antibodies for Wilms tumor 1 peptide greatly enhances therapeutic potential. Leukemia, 29: 2238-2247.

Zhao Q, Chan YW, Lee SS, Cheung WT. 2009. One-step expression and purification of single-chain variable antibody fragment using an improved hexahistidine tag phagemid vector. Protein Expr Purif, 68: 190-195.

Zhao Q, Feng Y, Zhu Z, Dimitrov DS. 2011. Human monoclonal antibody fragments binding to insulin-like growth factors I and II with picomolar affinity. Mol Cancer Ther, 10: 1677-1685.

Zhao Q, Zhu Z, Dimitrov DS. 2012. Yeast display of engineered antibody domains. Methods Mol Biol, 899: 73-84.

Zhu Z, Bossart KN, Bishop KA, Crameri G, Dimitrov AS, McEachern JA, Feng Y, Middleton D, Wang LF, Broder CC, Dimitrov DS. 2008. Exceptionally potent cross-reactive neutralization of Nipah and Hendra viruses by a human monoclonal antibody. J Infect Dis, 197: 846-853.

Zhu Z, Dimitrov AS, Bossart KN, Crameri G, Bishop KA, Choudhry V, Mungall BA, Feng YR, Choudhary A, Zhang MY, Feng Y, Wang LF, Xiao X, Eaton BT, Broder CC, Dimitrov DS. 2006. Potent neutralization of Hendra and Nipah viruses by human monoclonal antibodies. J Virol, 80: 891-899.

Zhu Z, Dimitrov DS. 2009. Construction of a large naive human phage-displayed Fab library through one-step cloning. Methods Mol Biol, 525: 129-142.

Zhu Z, Qin HR, Chen W, Zhao Q, Shen X, Schutte R, Wang Y, Ofek G, Streaker E, Prabakaran P, Fouda GG, Liao HX, Owens J, Louder M, Yang Y, Klaric KA, Moody MA, Mascola JR, Scott JK, Kwong PD, Montefiori D, Haynes BF, Tomaras GD, Dimitrov DS. 2011. Cross-reactive HIV-1-neutralizing human monoclonal antibodies identified from a patient with 2 F5-like antibodies. J Virol, 85: 11401-11408.

Zwick MB, Labrijn AF, Wang M, Spenlehauer C, Saphire EO, Binley JM, Moore JP, Stiegler G, Katinger H, Burton DR, Parren PW. 2001. Broadly neutralizing antibodies targeted to the membrane-proximal external region of human immunodeficiency virus type 1 glycoprotein gp41. J Virol, 75: 1089210905. 